\title{
Numerical Study of a Four-Roll Coating System*
}

\author{
Takeaki TSUDA** \\ ** Dai Nippon Printing Co., Ltd., Technology Development Center \\ 1-1-3 Midorigahara, Tsukuba, Ibaraki 300-2646, Japan \\ E-mail:Tsuda-T2@mail.dnp.co.jp
}

\begin{abstract}
The characteristics of a four-roll coating system were numerically investigated and compared with experimental data to validate the theoretical models used in this study. In the theoretical models, a film splitting model using a power-law-type equation, a roll-gap model based on elastohydrodynamics, and a flow model from a rotating-cylinder system were applied. The parametric computations for each operational condition revealed the steady and dynamic behaviors of a coating film and liquid films on the coating rolls. The results of the frequency response to the speed disturbances of the coating rolls indicated that the sensitivity of the lowest coating roll to the disturbance was half that of the others; this implies that the requirement for the accuracy of a driving system of the coating roll is not as severe as compared with others. The experimental data and the numerical results at steady state agreed well. Therefore, the theoretical models used in this research were found to be appropriate.
\end{abstract}

Key words: Numerical Analysis, Experiments, Film Flow, Roll Coating, Dynamic Response

\section{Introduction}

Designing the roll coating system is feasible without the complicated cavities for internal flows or the shapes of coating die lips, which are needed for slot or slide coating ${ }^{(1),(2)}$. Therefore, the system was widely used for the applications of traditional industrial materials, such as surface coatings for a paper or metal, adhesive coatings for multilayer flexible packages, and ink coating for printing systems ${ }^{(3)}$. The basic functions of a roll coating system for coating liquids ${ }^{(2)}$ are "feeding," "metering," "distributing," and "transferring onto the substrate." The number of coating rolls chosen is generally between two and six; the functions are allocated to each of the coating rolls for simplification of operations. Hence, it is important to examine the multiple-roll coating systems having multifunctions from the operational viewpoints.

Although the purpose of the roll coating operation is to obtain the desired coating thickness with adequate quality, it is difficult to control the coating thickness and the quality independently by means of the operational conditions of the system, such as rotating speeds of the coating rolls, coating gap between rolls, and web speed ${ }^{(4)}$. Therefore, the predictions of coating thickness from the operational conditions are very important for achieving favorable quality.

The multiple-roll coating introduced here can be investigated in three levels. First is the examination of the basic flows on a rotating roll as shown in Fig.1. While distributing the 
coating liquid to the next roll, the coating film on the roll acquires the rotating motion. Moffat $^{(5)}$ and Pukhnachev ${ }^{(6)}$ investigated the lubrication flow model of a rotating roll incorporating the effects of surface tension and gravity. Many scientists who followed their study revealed the complicated phenomena by improving the models ${ }^{(7)-(9)}$.

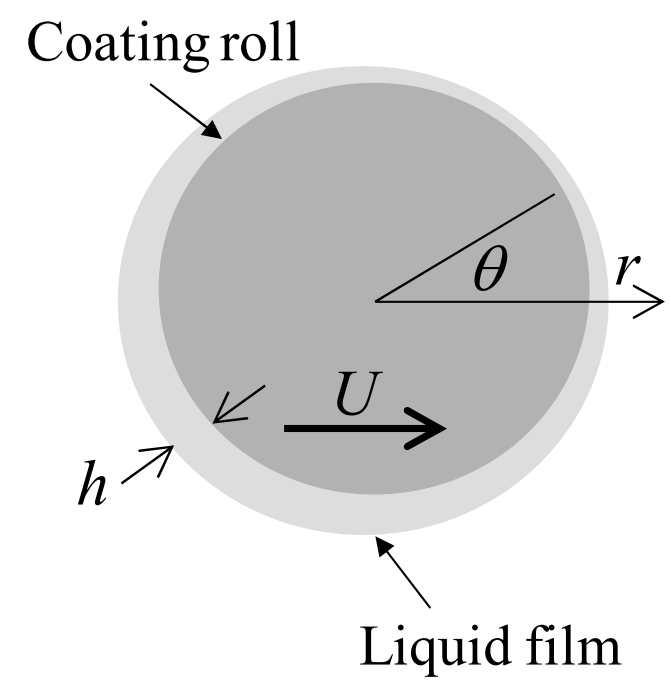

Fig. 1 Liquid film flow on a rotating roll

Second is the investigation of flow between two rolls as shown in Fig. 2. In the flow region, there are two problems to be solved for a roll coating system; one is a deformable gap treated as a lubrication model and the other is the flow distribution at a film splitting point. To address the deformable gap problem, the Dowson-Higginson's ${ }^{(10)}$ elastohydrodynamics lubrication (EHL) model provided a multivariable equation system to estimate the gap. Coyle ${ }^{(11)}$ pioneered the theoretical model of the deformable roll coating system by following the EHL approach. He proposed a simple deformable model using the Hookian spring model to obtain a more appropriate multivariable equation for roll coating system. Following this model, Carvalho and Scriven ${ }^{(12)}$ developed a two-dimensional model. Later, Carvalho ${ }^{(13)}$ introduced viscoelastic characteristics of the deformable roll and successfully predicted the realistic deformation of coating rolls.

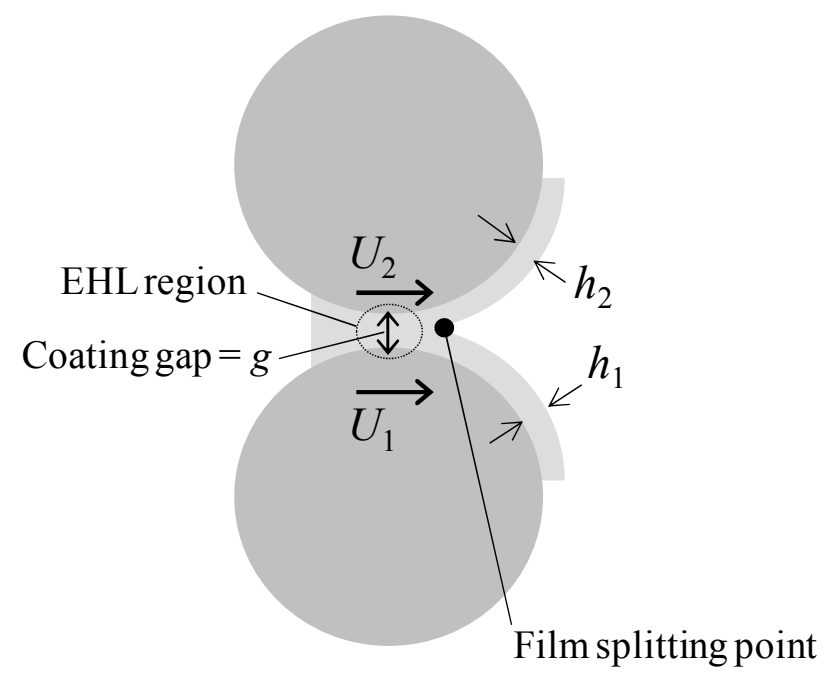

Fig. 2 Model of film splitting between two rolls 
For the problem of flow distribution at a film splitting point, many researchers attempted without success to derive an adequate lubrication-type equation to predict the film splitting ratio. Coyle found an accurate flow distribution at a film splitting point to solve a full Navier-Stokes equation using a finite element method ${ }^{(14)}$. Before Coyle's study, Benkreira $^{(15),(16)}$ experimentally obtained a simple power-law equation to predict the accurate film splitting ratio for Newtonian and non-Newtonian fluids, and Savage ${ }^{(17)}$ theoretically obtained an algebraic-type equation to predict the accurate film splitting ratio.

The third level for investigating a multiple coating system is by considering circulation flows between the coating rolls. Mill ${ }^{(18)}$ analyzed problems of steady/unsteady ink flow characteristics among fifteen rolls in order to minimize color density variation. Benjamin $^{(19)-(20)}$ analyzed transient/steady problems in the multiple-roll coating system using a time-lag model and conducted a variety of parametric studies in terms of operational conditions of the roll coating system. Tsuda ${ }^{(21)}$ analyzed steady-state of the four-roll coating system using algebraic equations that include the characteristics of the film splitting ratio, mass balance, and EHL effects.

As indicated by the previous studies, the multiple-roll coating system has complicated problems dealing with recirculation/recycle flows of the coating liquid in addition to the problems of coating flows on single or double rolls.

\section{Governing equations of roll coating system}

In this study, we will focus on a four-roll coating system, among the many complicated multiple-roll coating system used for industrial purposes. To understand the coating operations, we analyze the behaviors of liquid films on the rolls at "start-up," "splice motion," "steady state," and "adjustment of operational conditions." Benjamin ${ }^{(22)}$ elucidated that the prediction of a coating thickness using a lubrication model, neglecting the inertia effect, is accurate enough for practical purposes. Here, we examine the lubrication flow model, proposed by Hinch ${ }^{(8)}$. Our approach is the corrected version of Pubkhnachev's ${ }^{(6)}$ model using polar coordinates:

$$
\begin{aligned}
& \frac{\partial h}{\partial t}+\frac{\partial q}{\partial x}=0 \\
& q=U h-\frac{h^{3}}{3 \mu}\left\{\rho g_{v} \cos \left(\frac{x}{r}\right)-\frac{\partial p}{\partial x}\right\} \\
& p=\sigma\left\{\frac{\partial^{2} h}{\partial x^{2}}+\frac{h}{r^{2}}\right\}
\end{aligned}
$$

Here, $U, h, q, p, x, r, g_{v}, \sigma, \mu$, and $\rho$ indicate the rotational speed of the coating roll $(\mathrm{m} / \mathrm{s})$, the film thickness on the coating roll $(\mathrm{m})$, volumetric flow rate on the coating $\mathrm{roll}\left(\mathrm{m}^{2} / \mathrm{s}\right)$, pressure $(\mathrm{Pa})$, arc length on the coating roll $(\mathrm{m})$, the radius of the coating roll $(\mathrm{m})$, gravity $\left(\mathrm{m} / \mathrm{s}^{2}\right)$, surface tension of the coating liquid $(\mathrm{N} / \mathrm{m})$, viscosity of the coating liquid $(\mathrm{Pa} \cdot \mathrm{s})$, and density of the coating liquid $\left(\mathrm{kg} / \mathrm{m}^{3}\right)$, respectively. The equations are widely used and reliable in obtaining an appropriate result. To avoid numerical diffusion or instability from a convection term, we introduced the Kawamura-Kuwahara scheme ${ }^{(23)}$ of spatial discretization for a finite difference method as follows: 


$$
\frac{\partial q}{\partial x}=\frac{q_{i+2}-2 q_{i+1}+9 q_{i}-10 q_{i-1}+2 q_{i-2}}{6 d x}
$$

Here, $i$ indicates the node number in a spatial space. The second-order accuracy central-difference was used for other spatial derivatives. The Adams-Bashforth method of second-order accuracy was used for a temporal derivative.

We used an experimental equation based on the Dowson-Higginson equation, which can predict the coating gap between two coating rolls as follows:

$$
g=\alpha U_{m}^{0.7} P_{L}^{-0.13} R^{0.43}
$$

Here, $g, U_{\mathrm{m}}, \alpha, P_{\mathrm{L}}, R$, indicate coating gap between the rolls $(\mathrm{m})$, averaged roll speed $\left(\left(U_{1}+U_{2}\right) / 2\right)(\mathrm{m} / \mathrm{s})$, the experimental value, pressure between the rolls $(\mathrm{N} / \mathrm{m})$, and mean roll radius $\left(1 /\left(1 / R_{1}+1 / R_{2}\right)\right)(\mathrm{m})$, respectively. The gap $g$ is defined as the maximum gap that a liquid film can flow out. Therefore, in case a total flow rate $\left(U_{1} h_{1}+U_{2} h_{2}\right)$ does not reach the maximum flow rate $\left(g U_{\mathrm{m}}\right)$, we assume that the total flow can flow out from the gap. The gap model is used to obtain an accurate value using the experimental value and to avoid the effect of the temperature dependence of elasticity of rubber. The experimental value $\alpha$ is obtained using the relation $\left(U_{1} h_{1}+U_{2} h_{2}\right) / U_{\mathrm{m}}{ }^{1.7} / P_{L}{ }^{-0.13} / R^{0.43}$. Here, $h_{1}, h_{2}$ are experimental values measured by a laser displacement measurement system.

For the film splitting model, the power-law-type model proposed by Coyle ${ }^{(14)}$ and Benkreira $^{(15)}$ is used as follows:

$$
\frac{h_{1}}{h_{2}}=\left(\frac{U_{1}}{U_{2}}\right)^{2 / 3}
$$

Here, $h_{1}, h_{2}$ indicate liquid films $(\mathrm{m})$ on the rolls and $U_{1}, U_{2}$, indicate rotational speed of rolls $(\mathrm{m} / \mathrm{s})$, respectively. The index $2 / 3$ in the model is obtained from numerical and experimental methods in the Newtonian case ${ }^{(14),(15)}$.

The four-roll coating system consisting of these models is shown in Fig. 3. The sequence of flow analysis used in this research is explained using the figure. First, the coating liquid in the liquid bank between Roll 1 and Roll 2 flows toward the coating gap $g_{1}$ and is separated into two films $\left(h_{1}, h_{2}\right)$ through film splitting. Because liquid film $h_{1}$ returns to the liquid bank, we focus on liquid film $h_{2}$. Using equations (1)-(3) for the process occurring between $g_{1}$ and $\mathrm{g}_{2}$, the state of liquid film $h_{2}$ can be obtained. At the next gap $\mathrm{g}_{2}$, the total flow rate, adding liquid film $h_{5}$, is estimated by considering the maximum gap. The liquid film metered here is separated into two films $\left(h_{6}, h_{3}\right)$ through splitting. Liquid film $h_{3}$ is separated into $h_{5}$ and $h_{4}$ through film splitting at gap $g_{3}$. Considering the whole process, we can see that the liquid films affecting the coating thickness $h_{4}$ are $h_{2}, h_{3}$, and $h_{5}$.

To solve equations (1)-(3), we used a finite difference method with appropriate matching and boundary conditions at matching points $\left(\mathrm{MP}_{1-3}\right)$. In the numerical analysis, we used nodal number of finite difference and a Courant number of 400 and 0.005 , respectively. 
Matching point 1 Matching point 2 Matching point 3

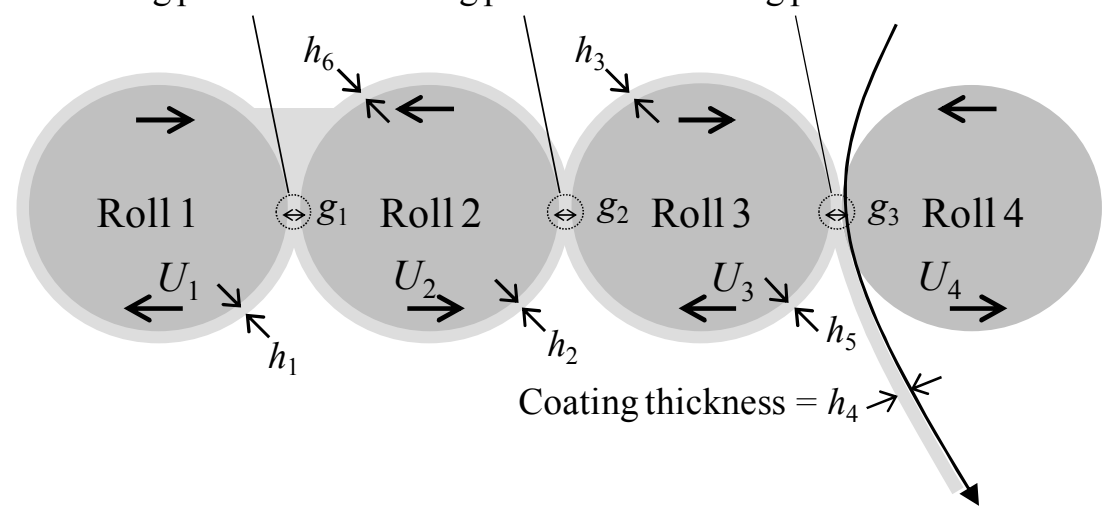

Fig. 3 Coating flow of four-roll coating system

For investigating the dynamic response of a multiple-roll coating system to a disturbance, we focus on the variation of roll speeds, which is the major source of disturbance in the system. In general, the coating rolls are driven by the torque of motors transmitted through couplings, belts, and reduction gears. Therefore, the speeds of the coating rolls tend to fluctuate due to misalignments, variation in control, and coating roll run-out. The variation in speeds can be defined as follows:

$$
U(t)=U_{\text {steady }}(1+\varepsilon \sin \varpi t)
$$

Here, $\varepsilon$ and $U_{\text {steady }}$ indicate the magnitude of disturbance of a coating roll speed and roll speed at steady state, respectively. In general, for high-precision industrial production the fluctuation must be maintained within a steady level of a few percentage points ${ }^{(24)}$. In this study, the magnitude of fluctuation is set at $1 \%$ for examining high-precision use.

In actual production, a splice motion, which switches an old roll of a substrate to a new roll, creates a disturbance in the roll coating system. In the splice motion, Roll 4 shown in Fig. 3 moves to the right to create a sufficient gap between it and Roll 3, thus avoiding damage to the coating rolls due to sandwiching of the substrate joint between the gaps. Therefore, during splice motion, Roll 3 cannot supply the substrate with the coating liquid. This condition can be expressed as follows:

During splice motion $\quad h_{4}=0$

Otherwise, $\quad h_{4}=h_{5}\left(\frac{U_{4}}{U_{3}}\right)^{2 / 3}$

Furthermore, as there are unavoidable disturbances in real production, an adjustment of the operational conditions should be included. These operations, especially, changing the roll speeds slightly, are not executed frequently. In this study, we tested the effects by setting a $20 \%$ deviation from the standard conditions.

The standard conditions set in this study are as follows. The roll radii $r_{1}, r_{2}, r_{3}=0.21 \mathrm{~m}$ and $r_{4}=0.16 \mathrm{~m}$. Liquid viscosity, surface tension, and density, $\mu=0.1 \mathrm{~Pa} \cdot \mathrm{s}, \sigma=50 \mathrm{mN} / \mathrm{m}$, 
and $\rho=1000 \mathrm{~kg} / \mathrm{m}^{3}$, respectively. The experimental value $\alpha=44.004 \times 10^{-6}$. The roll speeds $U_{1 \text { steady }}=2.3 \mathrm{~m} / \mathrm{s}, U_{2 \text { steady }}=9.3 \mathrm{~m} / \mathrm{s}, U_{3 \text { steady }}=9.7 \mathrm{~m} / \mathrm{s}$, and $U_{4 \text { steady }}=10.0 \mathrm{~m} / \mathrm{s}$. The load pressures on the coating gaps $P_{L 1 \text { (between Roll } 1 \text { and Roll 2) }}=4460 \mathrm{~N} / \mathrm{m}, P_{L 2 \text { (between Roll } 2 \text { and Roll } 3 \text { ) }}$ $=7477 \mathrm{~N} / \mathrm{m}$, and $P_{L 3 \text { (between Roll } 3 \text { and Roll 4) }}=8918 \mathrm{~N} / \mathrm{m}$.

\section{Computational analysis}

We compute the dynamic response of liquid films on each roll under various conditions of the four-roll coating system.

\subsection{Transient problems}

\subsubsection{Start-up characteristics}

Understanding the start-up characteristics of a roll coating system is important for achieving high yield rate in a production. The dynamic start-up responses of film thickness in each matching point of the rolls are shown in Fig. 4. Here, $h_{2}, h_{3}$, and $h_{5}$ are film thicknesses at $\mathrm{MP}_{1}, \mathrm{MP}_{2}$, and $\mathrm{MP}_{3}$, respectively. The coating thickness indicated here is the film thickness of $h_{4}$ at $\mathrm{MP}_{3}$. In the initial condition, there are no liquid films on any roll.

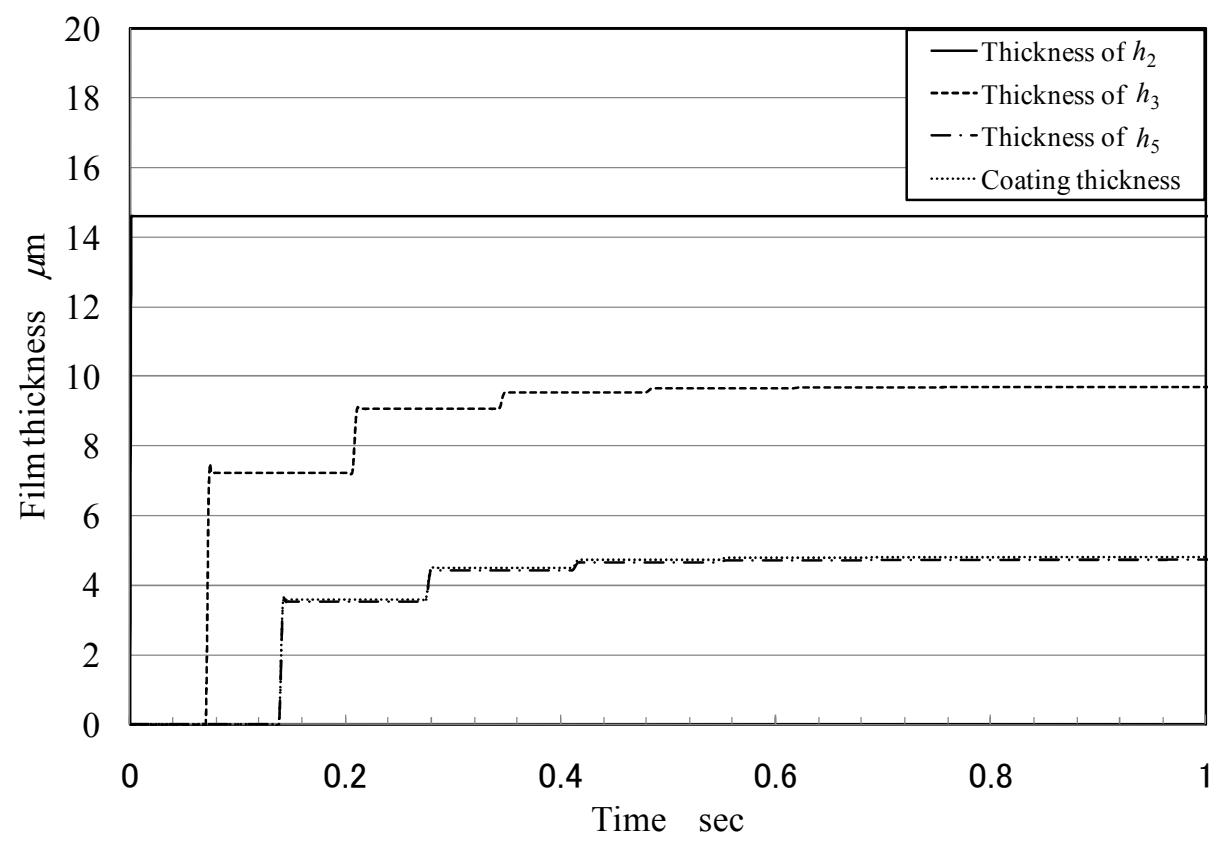

Fig. 4 Transient response of film thickness at start up

As shown in Fig. 4, an initial liquid film on a roll is supplied from $\mathrm{MP}_{1}$. Then it moves around the roll and is transferred to a next upper roll. In the operational conditions, it is found that coating film thickness $h_{4}$ attains a steady state in $0.5 \mathrm{~s}$. That is, although roll speeds reach set points immediately, about $5 \mathrm{~m}$ of start-up loss of the production will be created. To examine this problem, uniform precoating is done on the rolls. The dynamic responses of coating thickness $h_{4}$ with certain precoating conditions are shown in Fig. 5. 


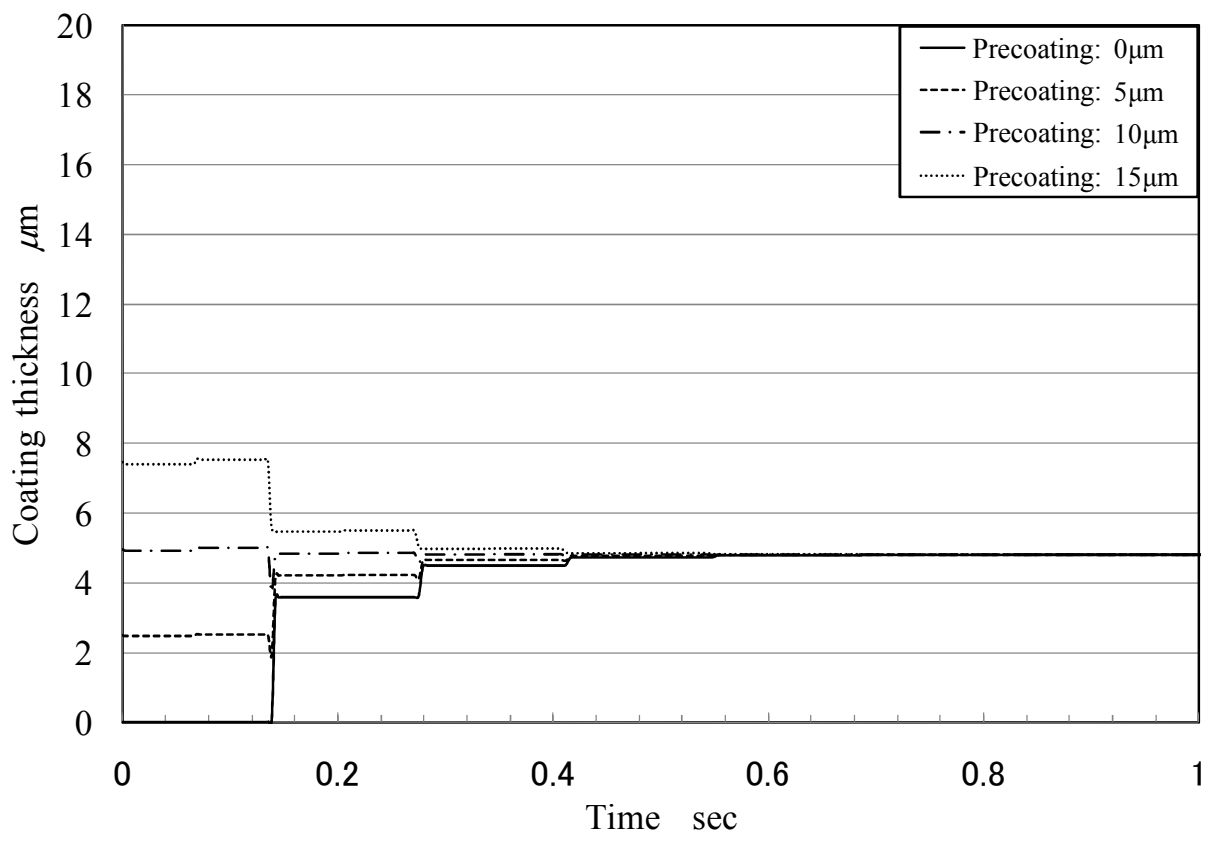

Fig. 5 Transient response of coating thickness at start up with precoating of rolls

As shown in Fig. 5, the time taken to reach a steady state decreases with increasing precoating thickness. This time is the least in a $10.0-\mu \mathrm{m}$ thick precoating, and it can be considered an optimum condition. Because excessive thickness of precoating film delays the time taken to reach a steady state, the optimum thickness value should be determined in each operational condition.

\subsubsection{Variation in operational conditions}

During production, it is possible that slight adjustment in the operational condition is made according to the change in material properties. Hence, it is important to analyze a process trend to maintain the quality of production. In this section, we compute the dynamic behavior of each film thickness on the rolls after a $20 \%$ adjustment in roll speed, to understand the characteristic of coating film recovery. The results are shown in Figs. 6-9. Here, the origin of a temporal axis indicates the coating starting point.

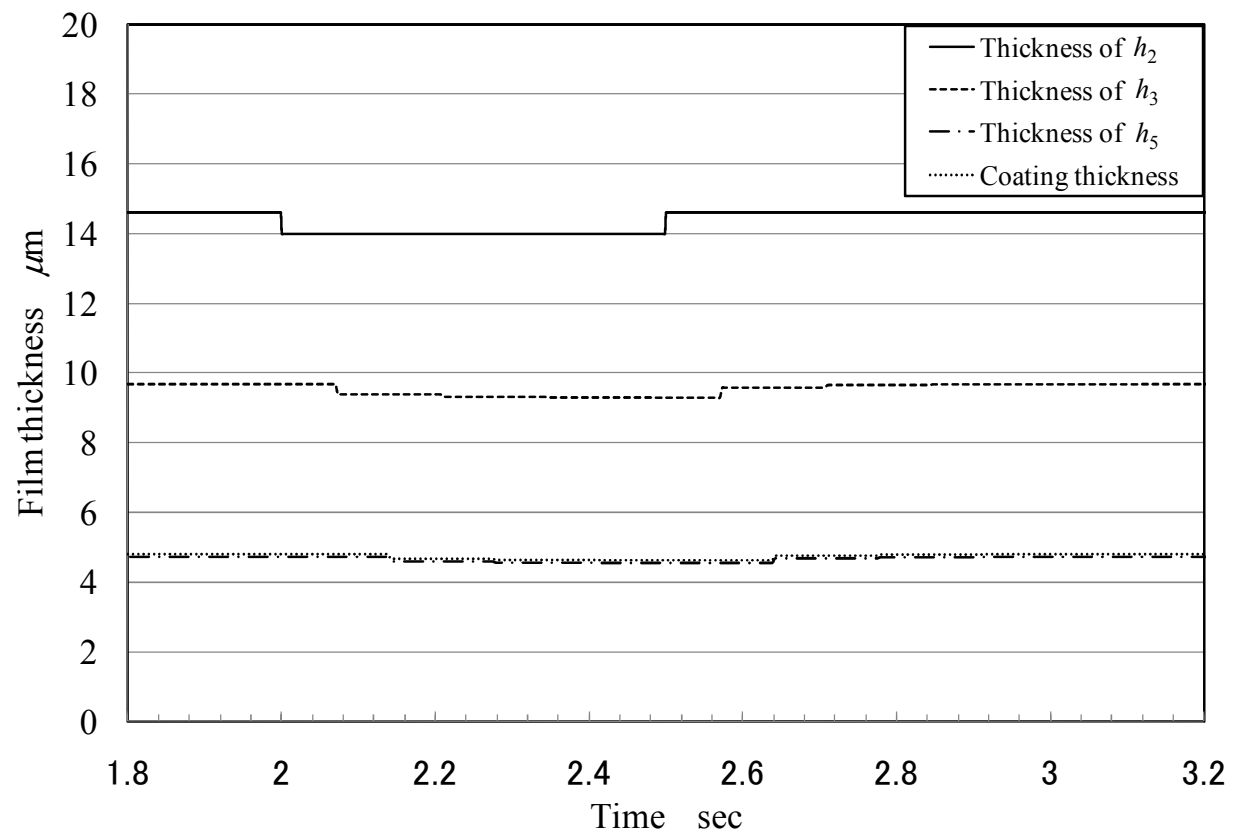

Fig. 6 Transient response with change in velocity of Roll 1 


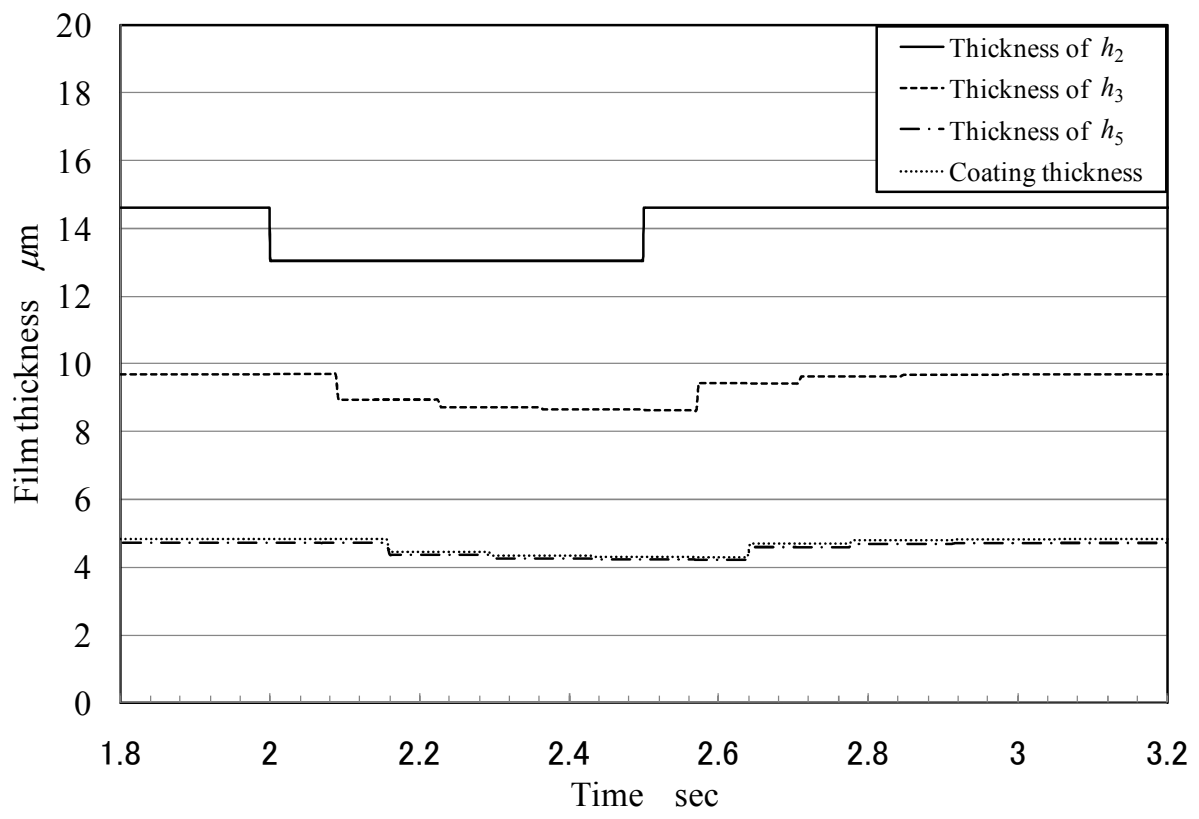

Fig. 7 Transient response with change in velocity of Roll 2

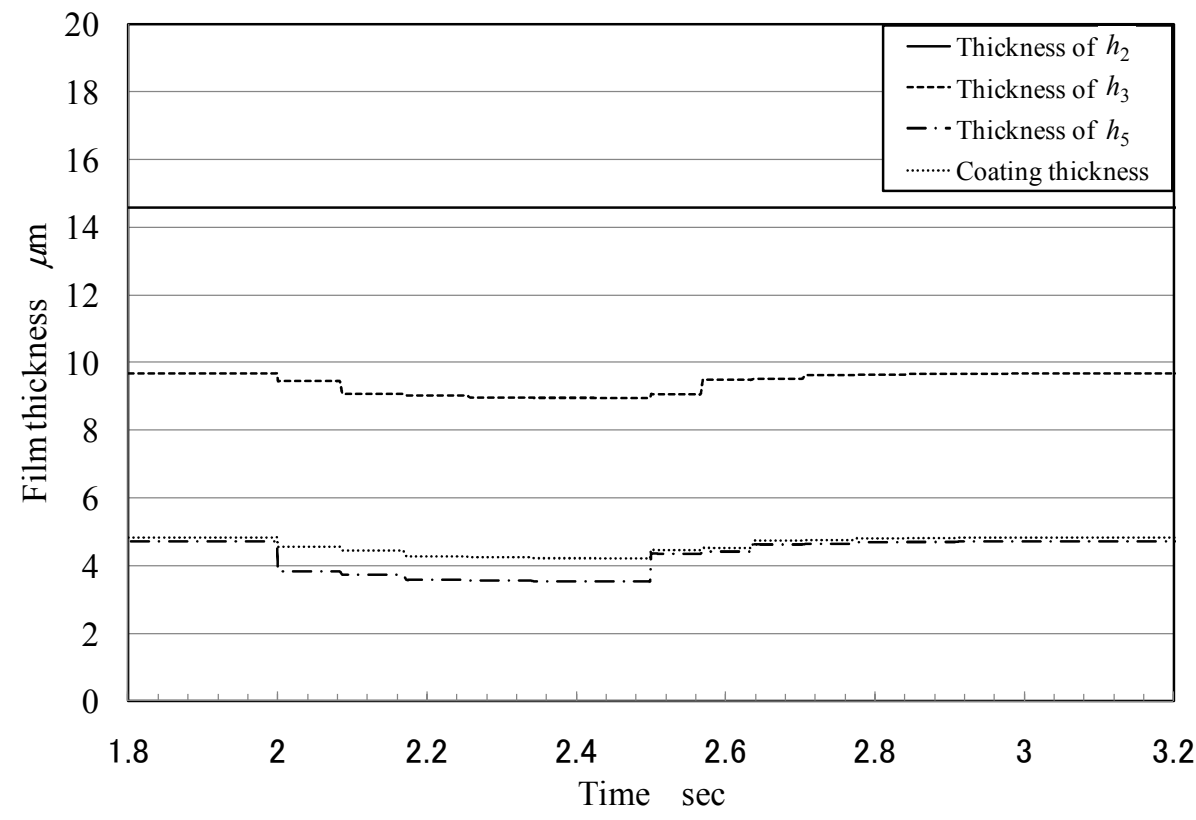

Fig. 8 Transient response with change in velocity of Roll 3 


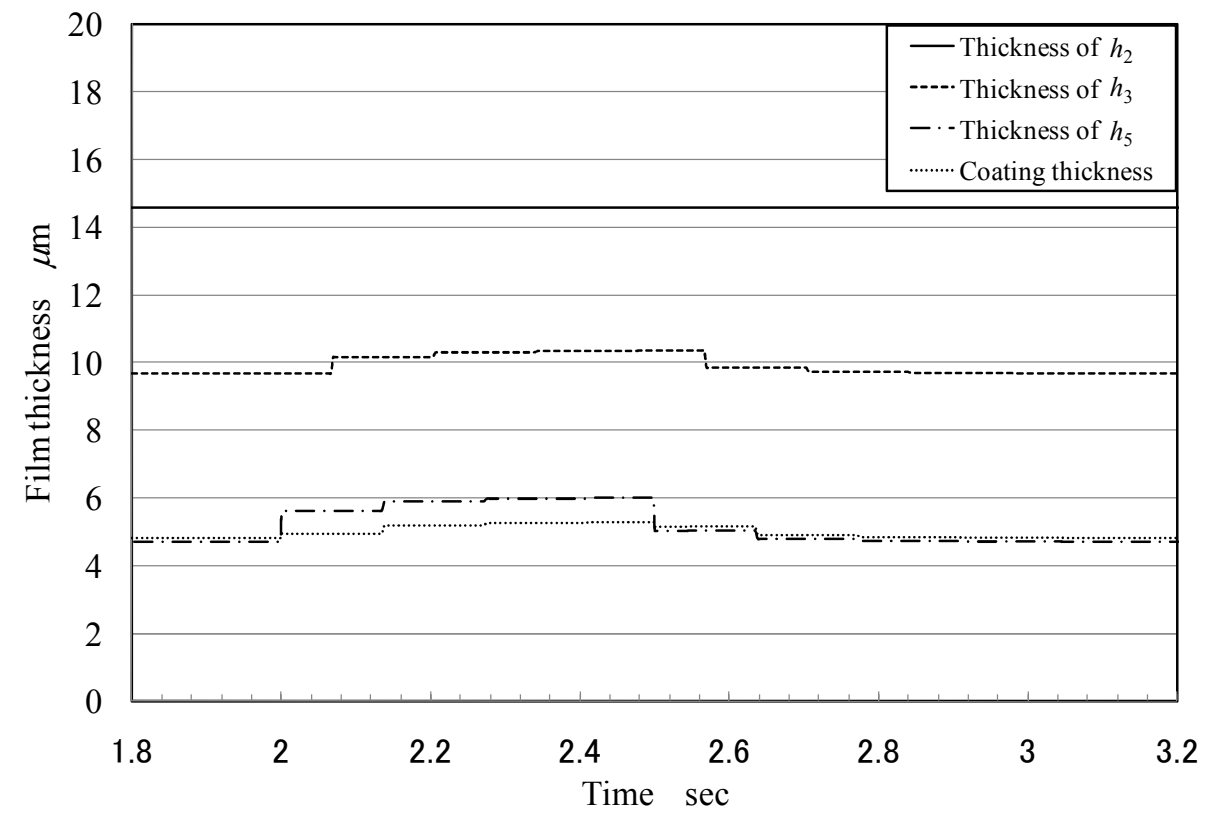

Fig. 9 Transient response with change in velocity of Roll 4

These results indicate that despite significant variations in liquid films on the rolls, the fluctuation of coating thickness is small and the time to reach a steady state is only $0.3 \mathrm{~s}$; it is same for liquid films on rolls in most cases. The only difference is that the coating film thickness increases with the $20 \%$ decrease in speed of Roll 4 . Because Roll 4 renders coating liquids to flow out into an external system, while other rolls circulate the coating liquids between the rolls, spillages of coating liquids are reduced by the decrease of speed. Thus, the volume accumulated in the internal system is considered to increase accordingly.

\subsubsection{Splice motion}

As described in Section 2, during splice motion, Roll 3 cannot supply the substrate with the coating liquid. It is important to immediately recover the coating quality in a post-splice process. In this study, the dynamic response of coating film thickness is computed to analyze the characteristics of transient behavior when a start-up ends and Roll 4 moves for 2-2.5 s after a complete steady state.

As shown in Fig. 10, during splice motion, coating liquids are not transferred to the substrate for $0.5 \mathrm{~s}$ although coating liquid is supplied by Roll 1 . Because the liquid is accumulated in the roll coating system, the liquid films on the rolls thickens rapidly. Moreover, for the accumulated volume on the rolls to flow out after splice motion, the same time as in splice motion is necessary to reach a steady state, as shown in Fig. 10. Although the relationship between a production loss and the splice motion has not been discussed so far, to minimize the loss, it is reasonable to prepare a quick splice motion system. When the splice motion duration is long, the film thickens on the rolls, and possible hazards, such as scattering of excess liquid into the surroundings due to the EHL condition, occur at every gap region. Hence, brief and accurate treatments for splice motion are essential. 


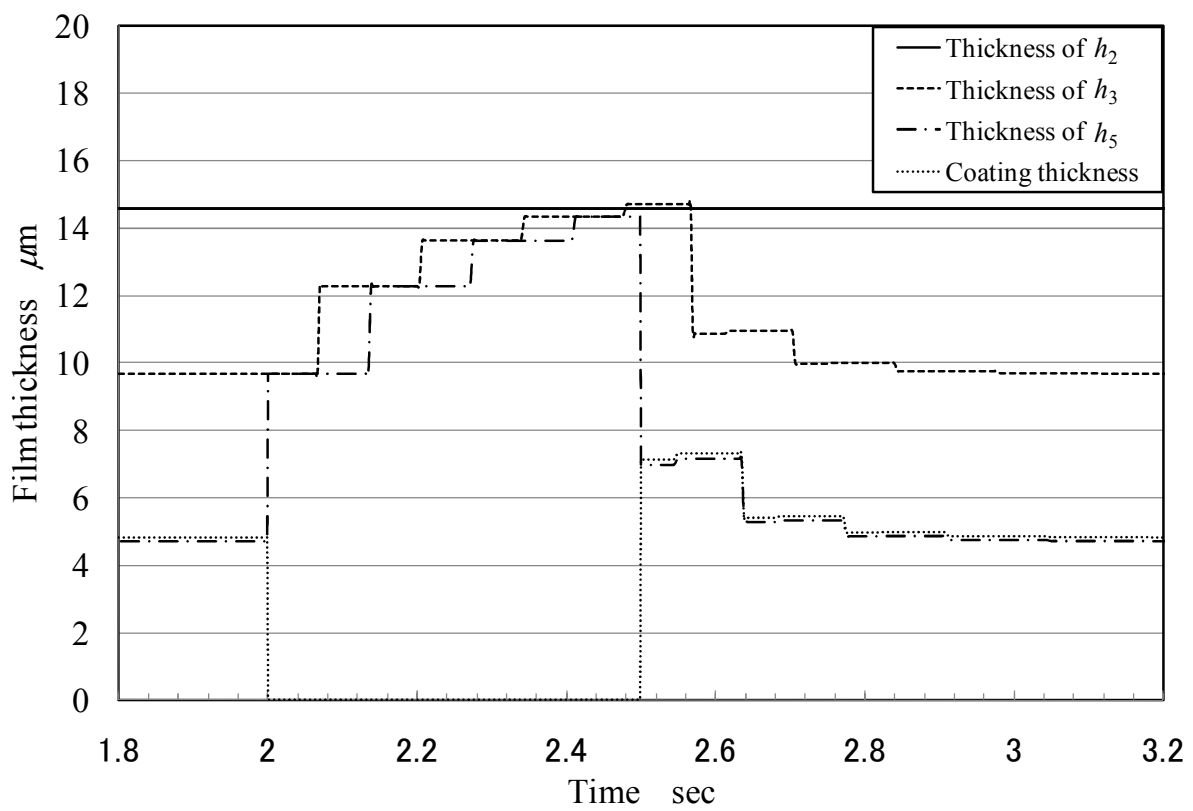

Fig. 10 Transient response in splice motion

\subsection{Steady state conditions}

Next, the parametric computations for each operational condition in a steady state are examined. We varied the conditions of the web speed, roll speed, and pressure load between the rolls and obtained the following results:

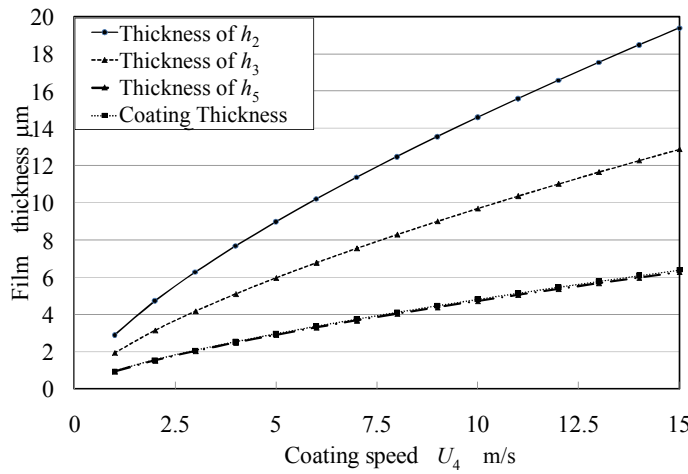

Fig. 11 Steady-state response with change in Coating speed $U_{4}$

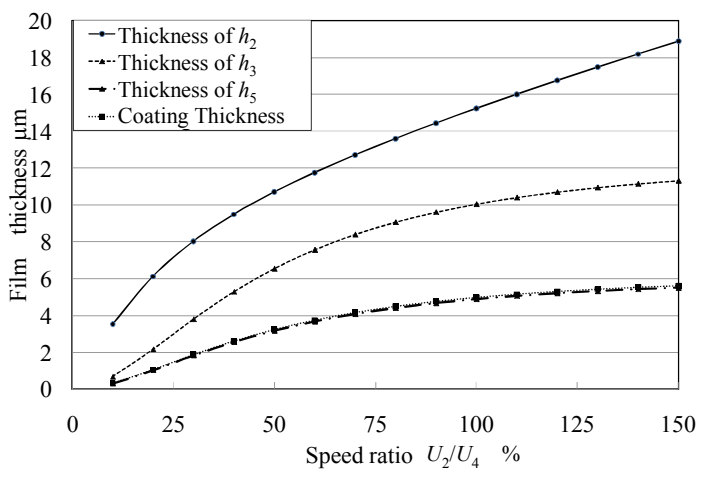

Fig. 13 Steady-state response with change in Roll 2 speed ratio $U_{2} / U_{4}$

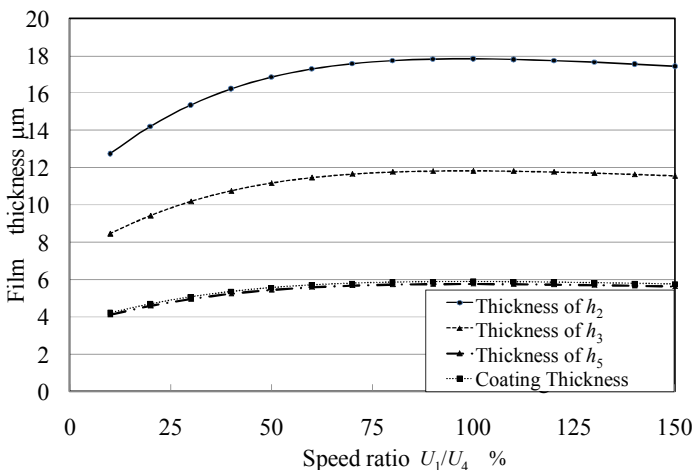

Fig. 12 Steady-state response with change in Roll 1 speed ratio $U_{1} / U_{4}$

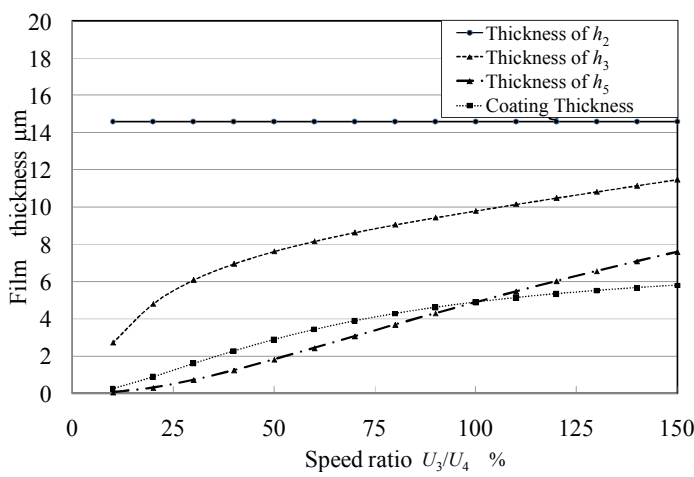

Fig. 14 Steady-state response with change in Roll 3 speed ratio $U_{3} / U_{4}$ 


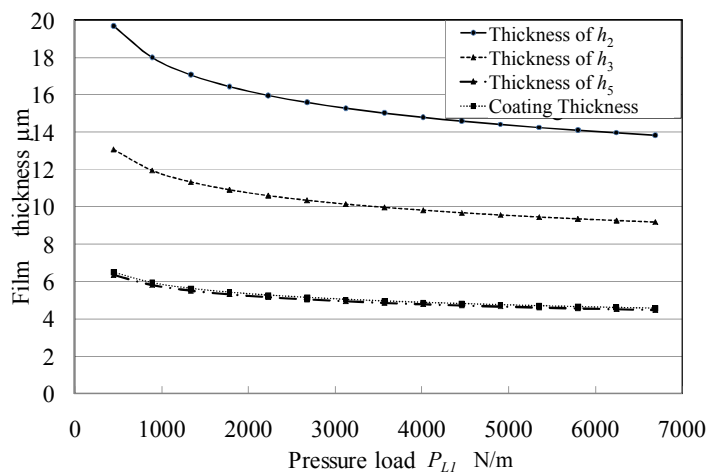

Fig. 15 Steady-state response with change in pressure load between Rolls 1 and 2

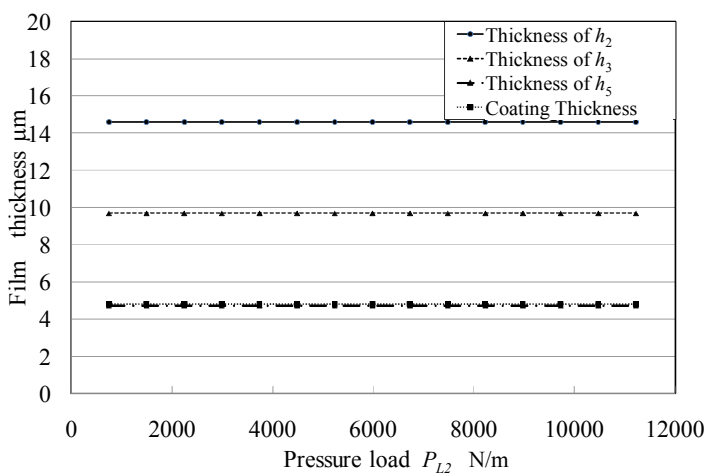

Fig. 16 Steady-state response with change in pressure load between Rolls 2 and 3

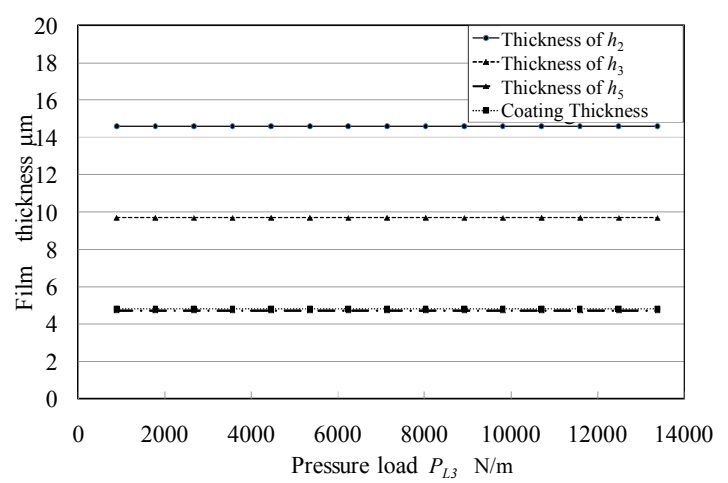

Fig. 17 Steady-state response with change in pressure load between Rolls 3 and 4

As shown in Fig. 11, with the increase in web speed, both the film thickness on the rolls and the coating thickness increases. The reason for this phenomenon is possibly because the expansion of $g_{1}$ by an EHL effect increases the flow rate given to the roll coating system. From Fig. 12, it is found that as the speed $U_{1}$ increases, the film thickness on rolls and coating thickness increase due to the EHL effect at $\mathrm{MP}_{1}$; however, when $g_{2}$ begins to expand due to the EHL effect, all of the liquid films consisting of $h_{2}$ and $h_{5}$ cannot penetrate the gap, and so the coating thickness reaches its maximum limit. When speed $U_{1}$ exceeds speed $U_{2}$, each film thickness begins to decrease because of the effect of film splitting. In Fig. 13, the same EHL effect at $g_{1}$ as in Fig. 12 can be observed. However, $g_{2}$ does not reach an EHL state because speed $U_{2}$ also increases. As shown in Fig. 14, in $U_{3}$ speed ratio less than $25 \%, g_{2}$ reaches an EHL state that enhances the increase in thickness $h_{3}$; however, in $U_{3}$ speed ratio more than $25 \%$, the effect of film splitting becomes dominant and causes a moderate increase in thickness $h_{3}$. Figure 15 shows an EHL effect of $g_{1}$ from consecutive increases in film thickness. In Figs. 16 and 17, it is found that $g_{2}, g_{3}$ do not reach an EHL state in the standard conditions.

\subsection{Frequency response}

The response sensitivity of coating thickness to periodic fluctuations in roll speeds is defined by GAIN $=\left|\left(h_{4}(t) / h_{4 \text { steady }}-1\right) / \varepsilon\right|$. The frequency responses of gain are shown in Figs. 18-21. 


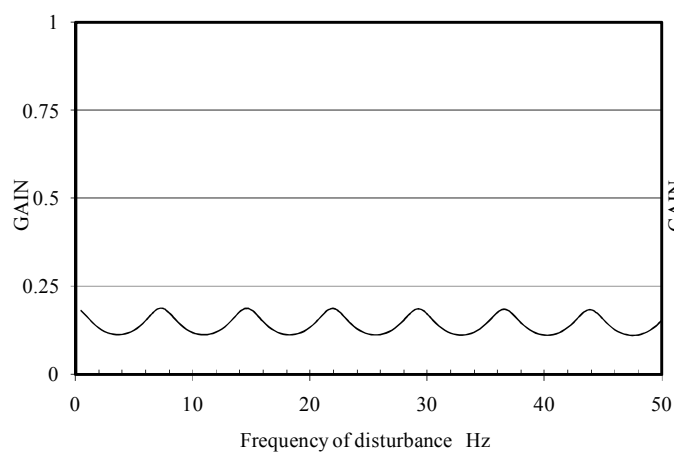

Fig. 18 Response to disturbance on Roll 1

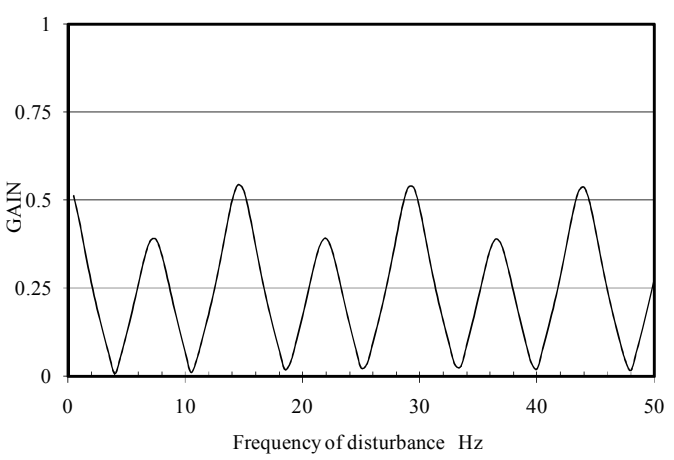

Fig. 20 Response to disturbance on Roll 3

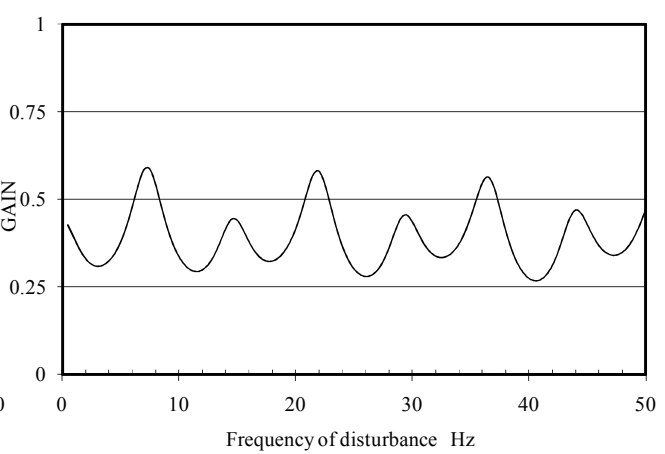

Fig. 19 Response to disturbance on Roll 2

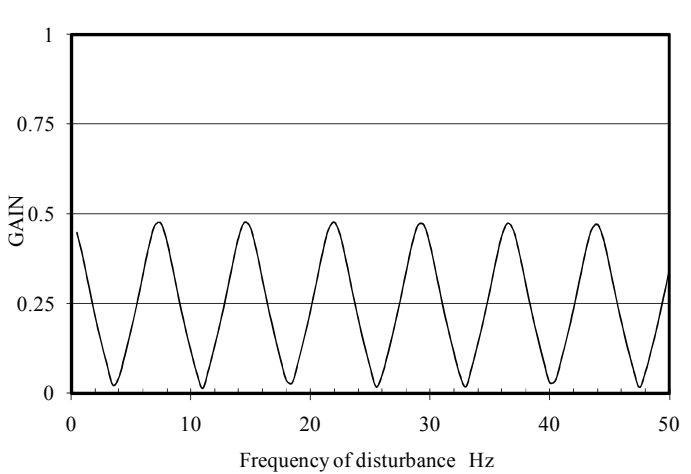

Fig. 21 Response to disturbance on Roll 4

Benjamin ${ }^{(20)}$ analyzed transient behavior using periodically varied roll speeds in limited cases; however, the frequency response of liquid films on rolls indicates that the coating stability characteristics were not present. Comparing all data, it was found that each condition of periodically varied roll speeds has unique properties like the response peak and trough appearing periodically in a frequency region.

From a practical viewpoint, it was found that the sensitivity of the lowest coating roll "Roll 1" to the disturbance was half that of the others, which implies that the requirement for the accuracy of a driving system for the coating roll is not as severe when compared with others. The reason the properties in the frequency region appear might be because the phase shift among perturbed films or between perturbed film and perturbed roll speed at $\mathrm{MP}_{2}$ or $\mathrm{MP}_{3}$ create the interference phenomena that exhibit a characteristic periodic response in a frequency region.

\subsection{Comparison with experimental results}

The experimental and computational results are compared as follows. The configuration of coating system, liquid properties, and other specifications are the same as the standard conditions. The coating thickness is measured by averaged coating weight in an area of $0.1 \mathrm{~m} \times 0.1 \mathrm{~m}$. The substrate and coating liquids used here are PET (Film thickness $=5.0$ $\mu \mathrm{m})$ and non-solvent type, respectively. The material of Roll 2 and Roll 4 is metal and the material of Roll 1 and Roll 3 is rubber (Hardness $=75^{\circ}$ ) covered metal. Each test sample was picked up carefully after sufficient warm-up coating. By carrying out the procedures, the repetition precision of the coating thickness was kept within $1.5 \%$. 


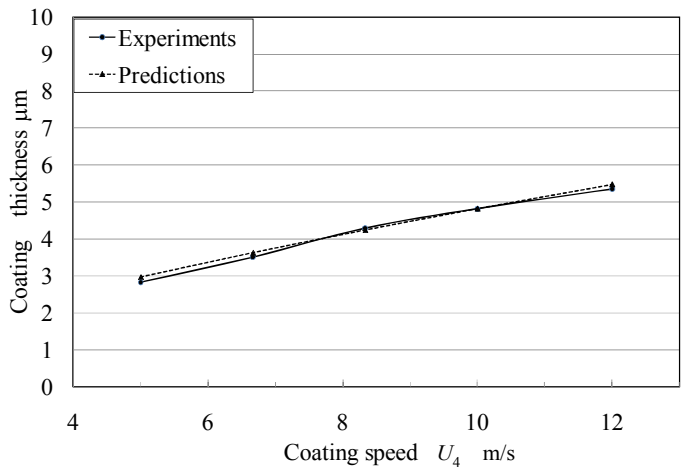

Fig. 22 Coating speed $U_{4}$

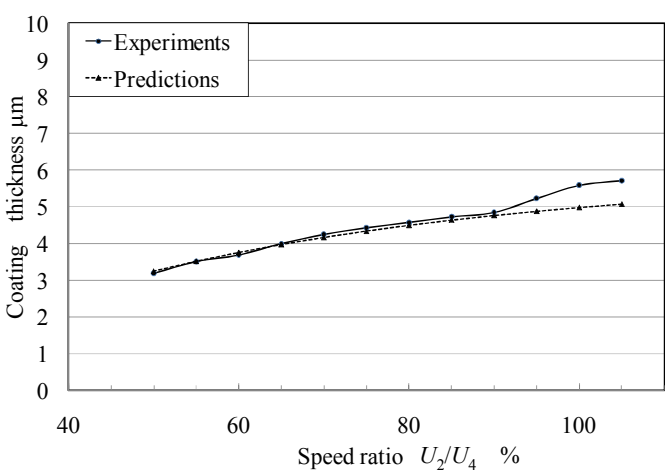

Fig. 24 Roll 2 speed ratio $U_{2} / U_{4}$
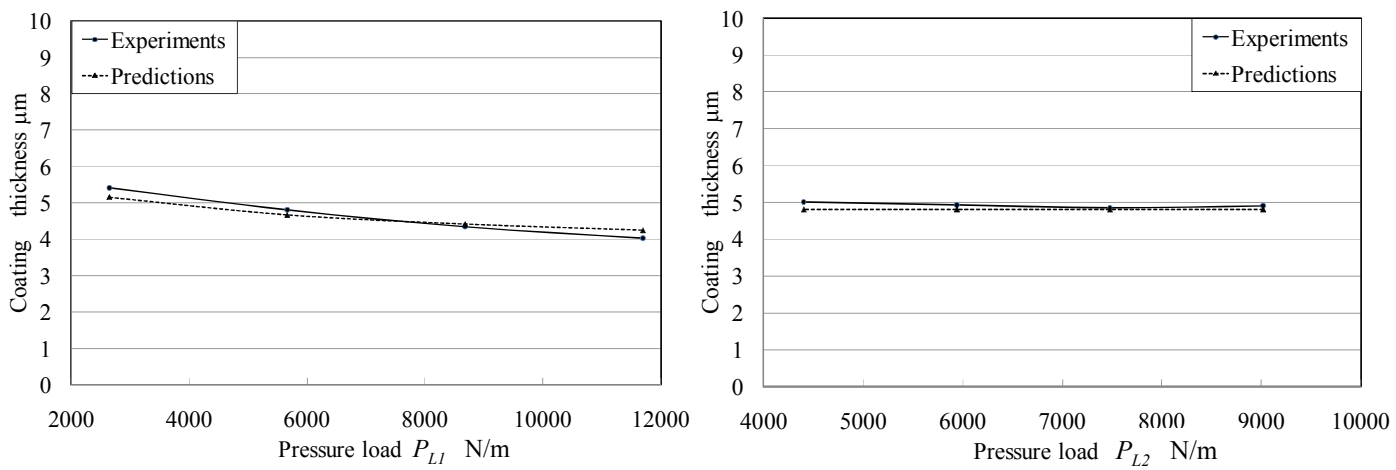

Fig. 26 Pressure load between Rolls 1 and 2 Fig. 27 Pressure load between Rolls 2 and 3

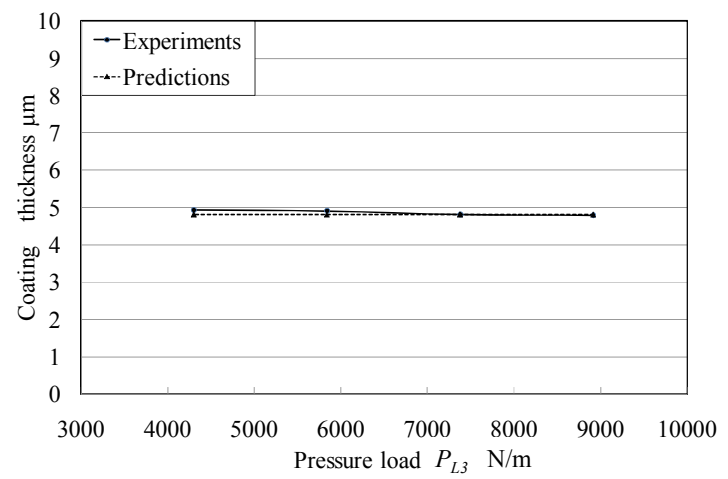

Fig. 28 Pressure load between Rolls 3 and 4

The difference in experimental and computational results shown in Figs. 24 and 25 can be considered from the unstable roll speeds caused by uncontrolled motors with the effects of friction in the gaps. In general, to avoid this problem, adjustments are made to control 
gains; however, the optimum gains were not found in this case. The magnitude of the observed variations of roll speed in each roll was less than a few percent around $0 \mathrm{~Hz}$. A total of the effects considering the sensitivities shown in Figs. 18-21 are insufficient to explain the phenomena. In addition, there is a possibility of varying the experimental value $\alpha$ by changing the gap friction. Since the partial difference in experimental and computational results is not negligible, the correction model including the actual properties of materials used in the coating process will be required to predict the coating thickness precisely.

\section{Conclusions}

In this study, the characteristics of a four-roll coating system were numerically investigated and compared with the experimental data to validate the theoretical models used. From the transient and frequency response analyses that reveal the dynamic behaviors of liquid films on coating rolls, the following conclusions can be drawn:

(1) From the dynamic numerical analysis, the behavior of a coating film and liquid films on the rolls is clarified. For the start-up problem, in particular, an optimum precoating thickness to minimize start-up loss was found.

(2) When splice motion periods are longer, the film thickens on the coating rolls, thus possibly causes the risk of scattering of liquid.

(3) It was found that the sensitivity of the lowest coating roll "Roll 1 " to the disturbance was half that of the others, which implies that the requirement for the accuracy of a driving system for the coating roll is not as severe as compared with others.

(4) The actual properties of materials used in the coating process will be required to predict the coating thickness precisely.

\section{Acknowledgment}

My deepest appreciation to Regents' Prof. Scriven of University of Minnesota, whose comments and suggestions throughout the course of my study were invaluable.

\section{References}

(1) Benjamin, D.F, Scriven L.E. and Colleagues, Coating flows: form and function, Industrial Coating Research, No.1 (1992), pp.1-37

(2) Benjamin, D.F, Scriven L.E. and Colleagues, Coaters analyzed by form and function, Industrial Coating Research, No.2 (1993), pp.1-31

(3) Harazaki, Y., New all of coating (in Japanese), (2009), Converting Technical Institute

(4) Schweizer, P. M. and Kistler, S. F., Liquid Film Coating, (1997), CHAPMAN \& HALL

(5) Moffatt, H. K., Behavior of a viscous film on the outer surface of a rotating cylinder, Journal de Mecanique, Vol.5 (1977), pp.651-673

(6) Pukhnachev, V. V., Motion of a liquid film on the surface of a rotating cylinder in a gravitational field, Z. Prikl. Mekh. Tekh. Fiz., No.3 (1977), pp.78-88

(7) Hansen, E. B. and Kelmanson, M. A., Steady, viscous, free-surface flow on a rotating cylinder, J. Fluid Mech., Vol.272 (1994), pp.91-107

(8) Hinch, E. J. and Kelmanson, M. A, On the decay and drift of free-surface perturbations in viscous, thin-film flow exterior to a rotating cylinder, Proc. R. Soc. Lond., A459 (2003), pp.1193-1213

(9) Evans P. L., Schwartz L. W., and Roy R. V., Three-dimensional solutions for coating flow on a rotating horizontal, cylinder: Theory and experiment, Physics of Fluids, Vol.17, No.7 (2005), pp. 072102.1-072102.20 
(10) Dowson D. \& Higginson G. R., Elasto-Hydrodynamic Lubrication, Chap.3 Lubrication of Rigid Cylinders, (1977), Pergamon Press, New York

(11) Coyle D. J., Forward roll coating with deformable rolls: a simple one-dimensional elastohydrodynamic model, Chem. Eng. Sci., Vol.43, No.10 (1988), pp.2673-2684

(12) Carvalho M.S. and Scriven L.E., Flows in Forward Deformable Roll Coating Gaps: Comparison between Spring and Plane-Strain Models of Roll Cover, Journal of Computational Physics, Vol.138, No.2 (1997), pp. 449-479

(13) Carvalho M.S, Effect of thickness and viscoelastic properties of roll cover on deformable roll coating flows, Chem. Eng. Sci., Vol.58, No.19 (2003), pp. 4323-4333

(14) Coyle D. J. ,Macosko C. W. \& Scriven L. E., Film-splitting flows in forward roll coating, J. Fluid Mech., Vol. 171(1986), pp. 183-207

(15) Benkreira, H., Edwards M. F., and Wilkinson W. L., Semi-empirical model of the forward roll coating flow of Newtonian fluids, Chem. Eng. Sci., Vol.36, No.2 (1981), pp. 423-427

(16) Benkreira, H., Edwards M. F., and Wilkinson W. L., Roll coating of purely viscous liquids, Chem. Eng. Sci., Vol.36, No.2 (1981), pp. 429-434

(17) Savage M. D., Mathematical models for coating processes, J. Fluid Mech., Vol.117 (1982), pp.443-455

(18) Mill C. C., An experimental test of a theory of ink distribution, Advanced in printing Science and Technology, Vol.1 (1961), pp.183-197

(19) Benjamin D. F., Anderson T. J., and Scriven L. E., Multiple roll systems: Steady-state operation, AIChE Journal, Vol.41, No.5 (1995), pp.1045-1060

(20) Benjamin D. F., Anderson T. J., and Scriven L. E., Multiple roll systems: Residence times and dynamic response, AIChE Journal, Vol.41, No.10 (1995), pp. 2198-2211

(21) Tsuda T., The predictions of coating thickness in four roll coating, Abstracts of the 30th autumn annual meeting of The Society of Chemical Engineers, Japan, (1997-9)

(22) Benjamin D. F., Roll coating flows and multiple roll systems, PhD Thesis University of Minnesota, (1994), Published by University Microfilms International, Ann Arbor, MI.

(23) Kawamura T. and Kuwahara K., Computation of high Reynolds number flow around a circular cylinder with surface roughness. AIAA Paper, No.84-0340 (1984)

(24) Tsuda T., Dynamic Response Analysis and Control of Slot Coating, Journal of Fluid Science and Technology, Vol.4, No.3 (2009), pp.735-745 